\begin{tabular}{ccc}
\hline & International Journal of Engineering \& Technology, $7(2.28)(2018) 200-203$ \\
SPC & International Journal of Engineering \& Technology \\
Website www.sciencepubco.com/index.php/IJET & Research paper \\
\hline
\end{tabular}

\title{
Robotic Teaching Aid for Disabled Children: A Sustainable Solution for Industrial Revolution 4.0
}

\author{
N. Zamin*, N. I. Arshad, N. Rafiey, A. S. Hashim \\ *Corresponding author E-mail: norshuhani@unimy.edu.my
}

\begin{abstract}
Many special needs children suffer from a common characteristics impairment which appear as disability to interpret social cues, fail to use joint-attention tasks as well as a failure in social gaze when communicating. This what makes them different than the normal children. The results of this difficulty are the special needs children often get frustrated when they are unable to expressively share their feeling and socially interact with the community. This research is investigating the problems faced by autistic, down syndrome and slow learner children to respond and communicate appropriately with the people around them and to propose an efficient approach to improve their social interaction. Malaysian education policy is to integrate students with learning difficulties or special educational needs. Thus, the development of a robotic approach using LEGO Mindstorms EV3 to aid the teaching and learning of special needs children especially autism in in Malaysia in introduced in this paper. Robotic approach in special education provides changes, inclusive and sustainable development of the disabled community towards supporting Industrial Revolution 4.0.
\end{abstract}

Keywords: Autism; Developmental Disabilities; Down Syndrome; Robotic; Slow Learner; Social Interaction; Special Education.

\section{Introduction}

Creating the abilities of the special needs children's day by day life is truly a test as each of them has diverse symptoms and is remarkable in their own particular ways. To help improve their life's quality, there are few real zones of education that must be thought to. Those ranges are communication, social and independence. Special needs children have an alternate route in learning and tolerating data that is different from normal children. The primary goal of this research is to enhance the teaching and learning experience of the special needs children from the fundamental methodologies and therapies. Many studies have previously proposed robotic approach as alternative therapy tool to improve social interaction skills and as well as reducing the emotional problem among the special needs children [2-4]. This is because robot has no feeling and can perform repetitive actions without getting bored or stressed. The proposed solution is to develop an LEGO robot to assist teachers, therapists as well as parents to improve social interaction skills among special needs children. This tool is not intended to replace the teachers and therapist but rather as an assistive tool.

\subsection{Case Study - Autism}

Autism is a complex neurobehavioral disorder that includes impairments in social interaction and developmental language and communication skills combined with rigid and repetitive behaviors [5]. Autism Spectrum Disorder (ASD) refers to the wide range of symptoms, skills and level of impairment or disability which include Asperger's and Kanner's Syndrome [6]. Among early signs of ASD is persistent deficit in social communication and interaction, repetitive patterns of behavior, interests or activi- ties and low ability in understanding multiple instructions. Typically, symptoms are presence in the first two years of a child's life [7]. Until today, the medical society is unable to confirm that the genetic factor is the main cause of ASD [8]. There is no specific medical treatment to cure autism, but many strategies and treatment options are available for autistic children [9]. Early diagnosis and correct therapy would help young children with autism to develop their full potential. Most current therapy methods aimed to improve the overall ability of the autistic children. As the number of children with autism has risen dramatically over the past couple of decades, experts have discovered that the earlier specialized therapy can be initiated; the outcome can be significantly improved [10]. The proposed approach is tested on autistic children at selected special schools and centers in Malaysia [11].

\section{Motivations}

Inspired by the difficulties of the observed current therapy methods and from the literature studies, a new and sustainable approach using robotic technology is proposed. The robotic intervention in nurturing autistic children has been very helpful in enhancing reading skills and generalizing knowledge for young pupils with autism. The sequence, progressive development is well defined and simple for therapists and parents to amend and keep track of the child's improvement. A rising comprehension of the robotic learning practice of autistic children is getting more attention from academic society. Autistic children go through their day by day activities by their weak senses that can be further enhanced with the aid of robotics [4]. It reduces the tension for them to rekindle what happens later, give a concise and clear path between actions, and aid them to be independent. The nonverbal signs 
shown by the robots to them can last a long time since they have a habit of repeating on every action they learnt [12].

Thus, robotic engagement has causes the evolution of education practices amongst autistic children. LEGO therapy is one of the current treatments for learning among disabled group of children including the autistic children. The LEGO therapy can improve cognitive development, creativity and hand-eye coordination while improving social skills when played together in a team [13, 14]. In this traditional LEGO therapy, children are normally supervised by assigned therapists. Our proposed method is to automate the LEGO therapy by using the LEGO Mindstorms, a programmable LEGO toolkit as a teaching and learning aid for the autism therapist. Our method is referred as the RoboTherapist that will adapt the ability to teach the basic foundation of knowledge through observation and hand-eye coordination with the supportive function from their attracted repetitive behaviours.

\section{Proposed Approach}

As the fourth industrial revolution (IR 4.0) and its embedded technology diffusion progress is expected to grow exponentially in terms of technical change and socioeconomic impact, we introduce a holistic approach that encompasses innovative and sustainable system solutions for special needs children [15]. In this article, a robot known as RoboTherapist using LEGO Mindstorms EV3 to teach autistic kids to differentiate shapes and encourage the kids to draw basic shapes correctly. It is a new approach and never been applied in special educations in Malaysia.

The RoboTherapist will be placed on a flat whiteboard and detect color by using color sensor that has been programmed in the LEGO Mindstorms EV3 Software. When the RoboTherapist detects the color on the whiteboard, it will start to draw shapes as pre-programmed. It will keep on looping until the user click end program. The association of colors and shapes are programmed as follows:

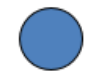

Circle

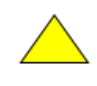

Square

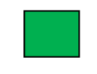

Triangle

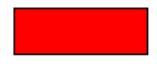

Rectangle
Fig. 1: The shapes and colors

The mechanism used by the RoboTherapist is the fixed rotation of the motor steering to draw each shape shown in Figure 1. The following figures illustrate the movement of RoboTherapist:

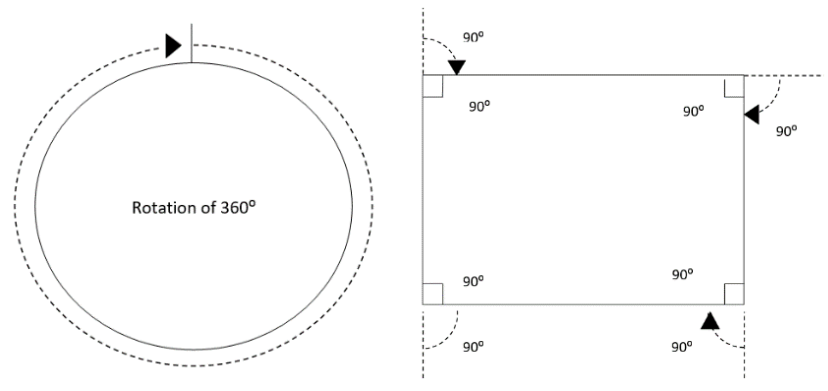

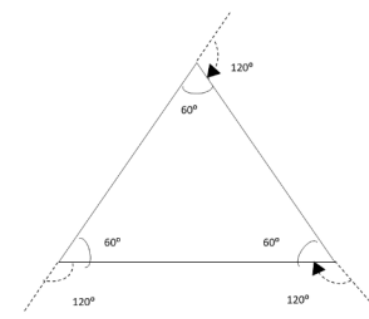

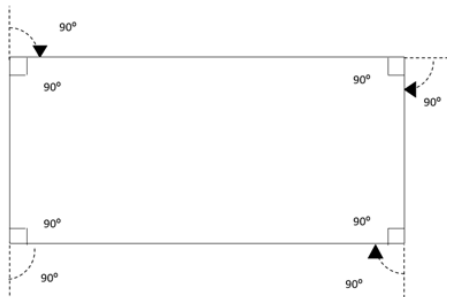

Fig. 2: The fixed motor directions

The flow chart in Figure 3 shows the flow of the overall program. RoboTherapist initiates by detecting the color read by the color sensor and draw the shapes as pre-programmed in Figure 2. Then, it will keep on looping until the stop button is pressed. The special needs children will observe the teaching from the RoboTherapist guided by the teachers. Their understanding is tested by a manual test designed to evaluate the effectiveness of the robotic approach.

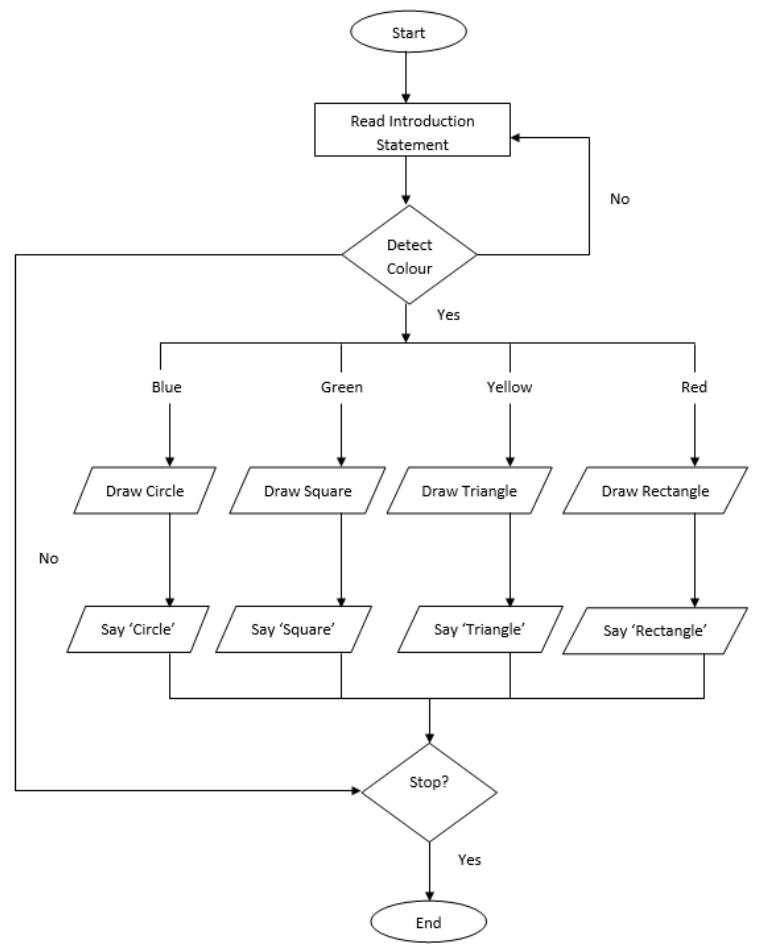

Fig. 3: RoboTherapist flowchart

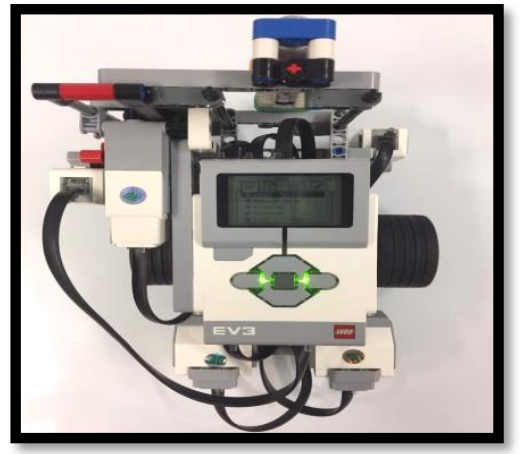

Fig. 4: RoboTherapist 


\section{Results and Discussions}

Initially, before the test was carried out, a pre-selection test was done to make sure whether the test candidates are fit for the test or otherwise. In the pre-selection, the potential candidates are asked to tinker with the RoboTherapist and their responses are recorded. If they can handle the robots well, then they are selected. This effort is highly crucial to avoid unnecessary damage on the robot by highly uncontrolled kids (a normal behaviour for some autistic children). Once selected, they will seat for the actual test where the therapist will assist the children to RoboTherapist. The comparative results between the traditional learning method and the robotic approach are presented. From the survey conducted we can see that most of the children with autism will get easily distracted, need to repeat several times in making them understand It is very challenging in attracting and retaining the autistic children attention, especially in learning.

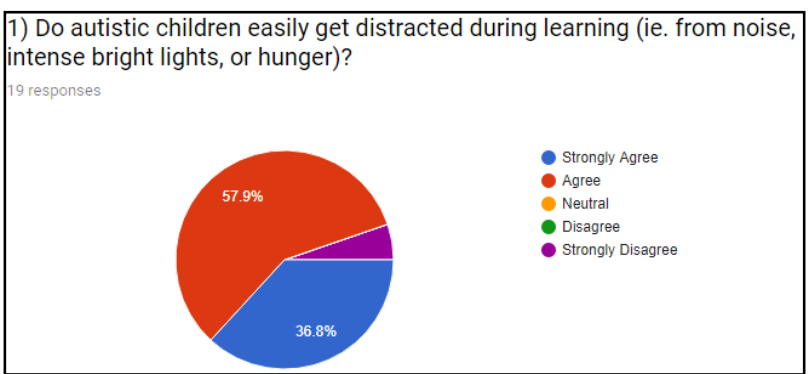

2) When teaching autistic children, do you think that we need to repeat several times to ensure that they understand?

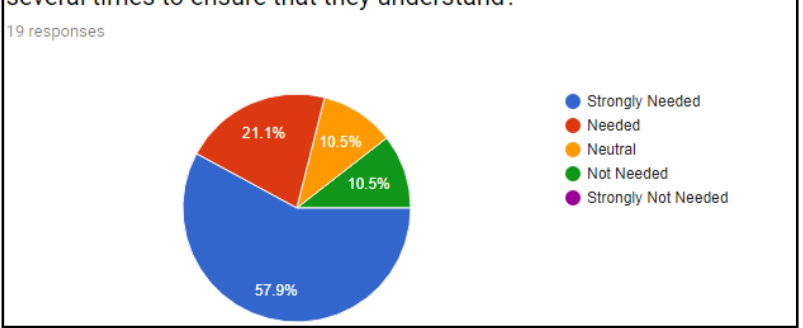

3) Do you find it challenging to teach and retain the autistic children's attention during school?
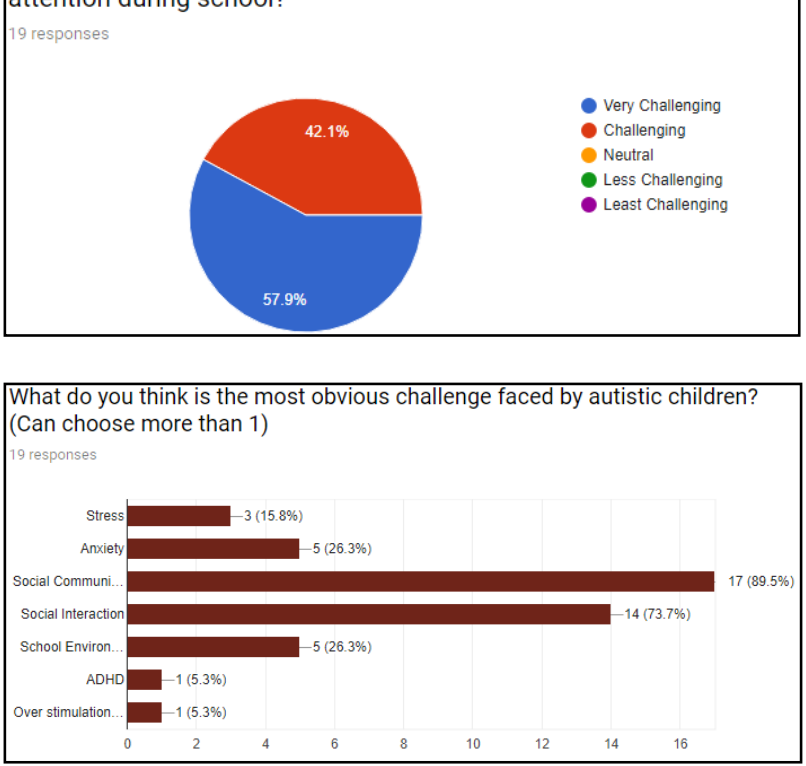

Fig. 5: Survey on students' attentiveness

We can also conclude that most of the major challenge in teaching the autistic children falls under 'Social Communication' where the children find it hard in letting people to control their emotion and behaviour. This happens because for them it is difficult to under- stand and follow the instructions given by the teachers. Then, the survey continues with the benefits of implementing or introducing the learning method with robot as a medium in teaching the autistic children. As we can see and observe from the results below, most of the respondents agree with the implementation of Robot in teaching basic shapes to the autistic children.
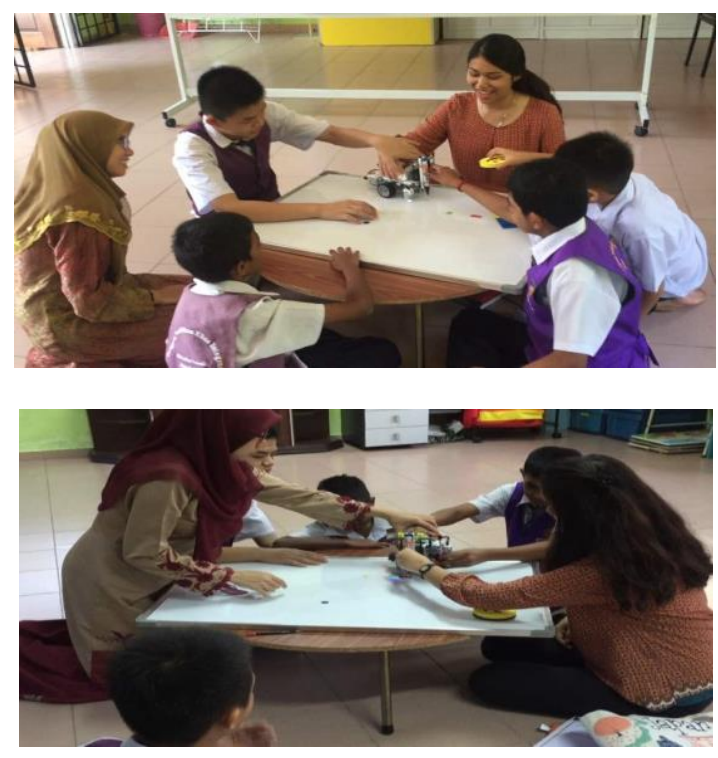

Fig. 6: Hands-on learning using robotic

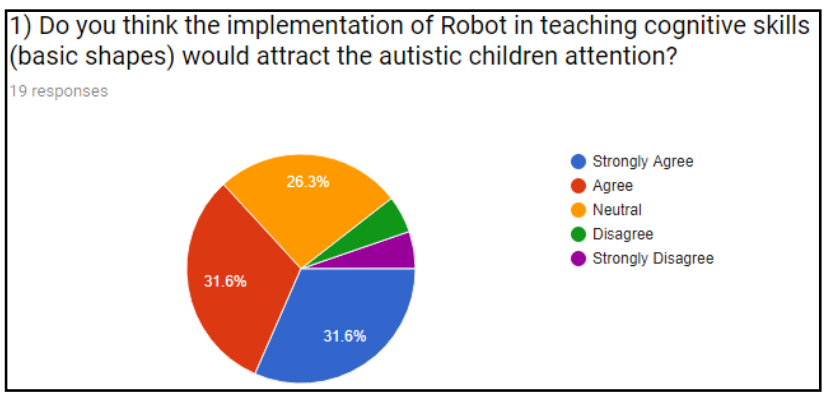

2) Do you think learning basic shapes with Robot would be beneficial to the autistic children?
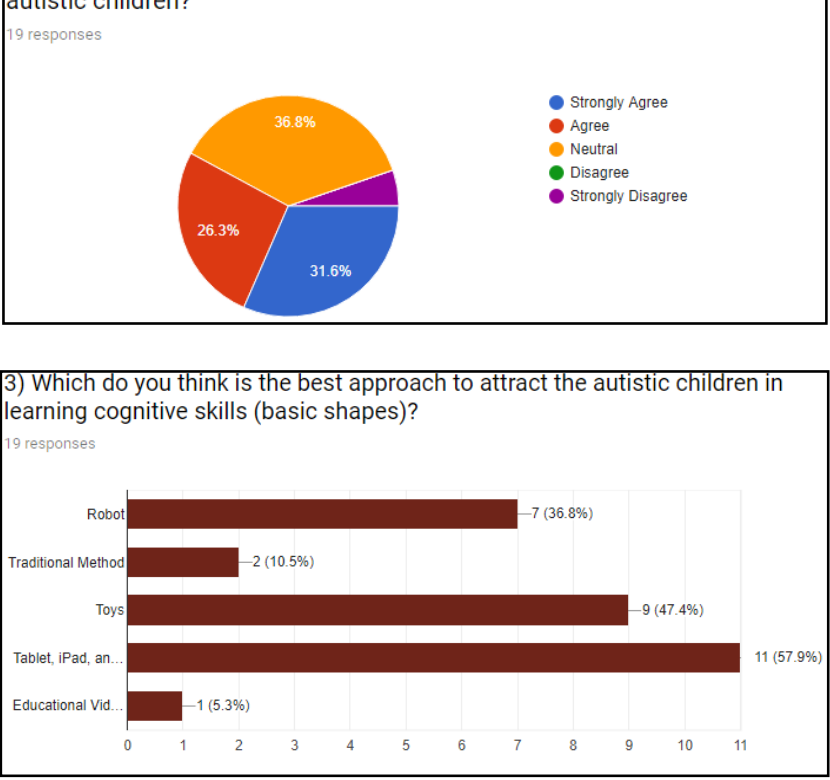

Fig. 7: Survey on the effectiveness of robotic approach 
Most of the respondents agreed that the teaching approach using robot is the best assistive tool for teaching the autistic children. In addition, below are the opinions shared by the adult respondents (teachers, parents and caretakers) throughout the survey. As the autistic children can easily get distracted therefore more attention are needed when teaching them. Thus, with the new teaching and therapy method by introducing the robot to the autistic children, attracts the children's attention and making learning basic shapes exciting and easy.

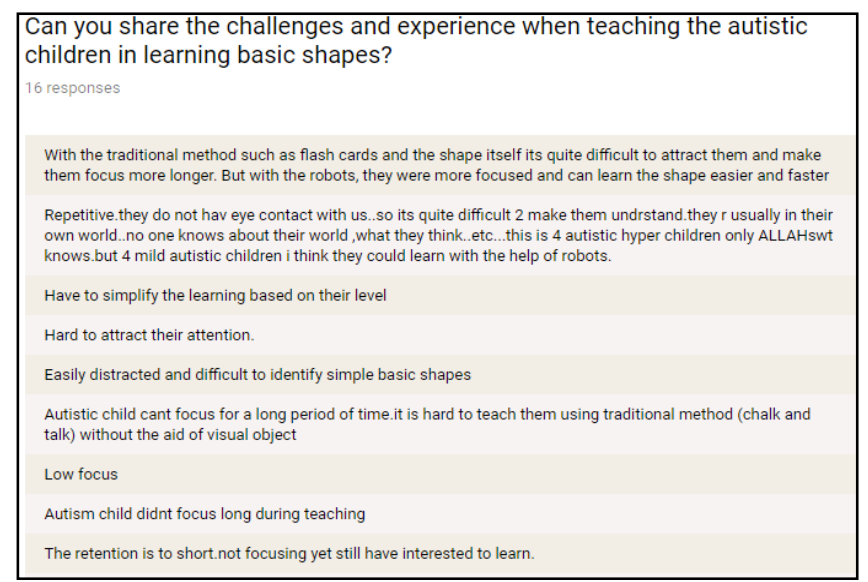

Fig. 8: Opinions on robotic approach

\section{Conclusion}

Robotic approach is a sustainable approach for teaching and learning. It encourages active learning with high attentive rate of the learners especially from the disabled community. This research shows how the learning of basic shapes can be made fun, easy and efficient for the special needs children. This project is to support the Social Innovation initiatives of the Malaysian government in the Eleventh Malaysia Plan. Social Innovation is parked under the 8th strategic plan which is to re-engineering economic growth for greater prosperity.

\section{References}

[1] M. Z. Ghani and A. C. Ahmad, "Stress among Special Education Teachers in Malaysia," Procedia - Social and Behavioral Sciences, vol. 114, pp. 4-13, 2014

[2] C. A. G. J. Huijnen, M. A. S. Lexis, R. Jansens, and L. P. d. Witte, "How to Implement Robots in Interventions for Children with $\mathrm{Au}$ tism? A Co-creation Study Involving People with Autism, Parents and Professionals," Journal of Autism Development Disorder, vol. 2017, pp. 3079-3096 2017.

[3] IESD, "IESD Case Study: Children on the Autism Spectrum Show Improvement with ROBOTS4AUTISM in Spartanburg, South Carolina," Interactive Educational Systems Design Inc., South Carolina2016.

[4] A. B. Martelo, "Social Robots to enhance therapy and interaction for children: From the design to the implementation "in the wild"," Doctor of Philosophy, ETSEEI LA SALLE - Electrònica, Universitat Ramon Llull, 2017.

[5] R. Singhania, "Autistic Spectrum Disorder," Indian Journal of Pediatrics, vol. 72, pp. 343-352, 2005.

[6] C. M. Murphy, E. Wilson, D. M. Robertson, C. Ecker, E. M. Daly, N. Hammond, et al., "Autism spectrum disorder in adults: diagnosis, management, and health services development," Neuropsychiatr Disease \& Treatment, vol. 12, pp. 1669-1686, 2016.

[7] S. Ozonoff, A. M. Iosif, F. Baguio, I. C. Cook, M. M. Hill, T. Hutman, et al., "A prospective study of the emergence of early behavioral signs of autism," Journal of the American Academy of Child \& Adolescent Psychiatry, vol. 49, pp. 256-266, 2010.

[8] I. Joshi, M. Percy, and I. Brown, "Advances in Understanding Causes of Autism and Effective Interventions," Journal on Developmental Disabilities, vol. 9, pp. 1-27, 2002.
[9] M. DeFilippis and K. D. Wagner, "Treatment of Autism Spectrum Disorder in Children and Adolescents," Psychopharmacol Bulletin, vol. 46, pp. 18-41, 2016.

[10] M. o. E. MoE, "Data Pendidikan Khas 2016," Ministry of Education Malaysia, Kuala Lumpur2017.

[11] A. Mulligan, R. J. Anney, M. O’Regan, W. Chen, L. Butler, M. Fitzgerald, et al., "Autism symptoms in attentiondeficit/hyperactivity disorder: a familial trait which correlates with conduct, oppositional defiant, language and motor disorders," Journal of autism and developmental disorders, vol. 39, pp. 197-209, 2009.

[12] F. Michaud and C. Théberge-Turmel, "Mobile robotic toys and autism," in Socially Intelligent Agents, ed Boston, MA: Springer, 2002.

[13] D. B. LeGoff, "Use of LEGO as a Therapeutic Medium for Improving Social Competence," Journal of Autism and Developmental Disorders, vol. 34, pp. 557-571, 2004.

[14] G. Owens, Y. Granader, A. Humphrey, and S. Baron-Cohen, "LEGO® therapy and the social use of language programme: An evaluation of two social skills interventions for children with high functioning autism and Asperger syndrome," Journal of autism and developmental disorders, vol. 38, p. 1944, 2008.

[15] R. Morrar, H. Arman, and S. Mousa, "The Fourth Industrial Revolution (Industry 4.0): A Social Innovation Perspective," Technology Innovation Management Review, vol. 7, pp. 12-20, 2017. 